\title{
COVID-19 y enfermedades autoinmunes: ¿población con alto riesgo para desarrollar enfermedad severa?
}

\section{COVID-19 and autoimmune diseases: population at high risk of developing severe disease?}

Correspondencia Ernesto Zavala Flores ernestozav123@gmail.com

Recibido: $11 / 07 / 2020$ Aprobado: $27 / 08 / 2020$

Citar como: Zavala-Flores E, Salcedo-Matienzo J, Berrocal-Kasay A. COVID-19y enfermedades autoinmunes: ¿población en alto riesgo para desarrollar enfermedad severa?. Acta Med Peru. 2020;37(3):407-9. doi: https://doi.org/10.35663/ amp.2020.373.1046

\author{
Ernesto Zavala-Flores ${ }^{1, a}$, Jannin Salcedo-Matienzo ${ }^{2, a}$, Alfredo Berrocal- \\ $\operatorname{Kasay}^{1,2, a, b}$ \\ ${ }^{1}$ Servicio Inmuno-Reumatología, Hospital Cayetano Heredia. Lima, Perú. \\ ${ }^{2}$ Facultad de Medicina Humana, Universidad Peruana Cayetano Heredia. Lima, Perú. \\ a Médico cirujano; ${ }^{b}$ especialista en Reumatología.
}

Señor editor,

La pandemia por la infección del virus SARS-CoV-2, ha tenido un fuerte impacto en la salud pública, economía y estilo de vida de la población en general. A la fecha, se reportan millones de infectados y más de quinientos mil muertes en todo el mundo, y estas cifras estarían en aumento. Desde el inicio de la pandemia, se intensificaron los esfuerzos para identificar los factores de riesgo para el desarrollo de la forma severa de la COVID-19. Se ha reportado que la diabetes, hipertensión arterial, obesidad, edad > 60 años, son algunas de las principales condiciones que están relacionadas al desarrollo del cuadro severo de la enfermedad ${ }^{[1]}$.

Una de las interrogantes es en relación a los pacientes con enfermedades autoinmunes y su tratamiento inmunomodulador. Se ha planteado la posibilidad que, dada la condición de inmunosupresión farmacológica, se verían más afectados e inclusive podrían desarrollar la forma severa de la COVID-19. Sin embargo, a la fecha son muy limitados los reportes que demostrarían este hecho.

Debido a esto, se realizó una búsqueda de la literatura publicada desde el 1 de marzo hasta el 20 de agosto de 2020 en PubMed y Google Académico, las palabras clave utilizadas fueron "COVID19", "autoimmune disease", "SARS-CoV-2", "risk factor", "comorbidities", "coronavirus disease 2019", "nCoV-2019", "enfermedades autoinmunes". Se encontró un total de 11 estudios de pacientes con enfermedades autoinmunes e infección por SARS-CoV-2; en la Tabla 1 se describen las características de las series publicadas.

Se observó un total de 977 pacientes con los diagnósticos planteados, de los cuales 631 fueron hospitalizados. Las patologías más frecuentes fueron artritis reumatoide (211 pacientes), lupus eritematoso sistémico (96 pacientes) y artritis psoriásica (70 pacientes); las comorbilidades más frecuentes fueron: hipertensión arterial, diabetes mellitus y enfermedades cardiovasculares. Del total, se reportó 116 fallecidos (11,9\%), es importante considerar que las comorbilidades antes descritas contribuirían a la mortalidad por COVID-19 más no la enfermedad autoinmune en sí, como es descrito por otros autores ${ }^{[2]}$.

Es evidente que al momento actual, no se puede afirmar que los pacientes con enfermedades autoinmunes presenten «menor riesgo» de desarrollar la forma severa de la COVID-19; sin embargo, está claro que en los diferentes estudios epidemiológicos publicados no se describe que esta condición sea un factor de riesgo independiente para el desarrollo del cuadro severo o de mortalidad por COVID-19 [3,4]. 
Tabla 1. Series de casos de enfermedades autoinmunes y COVID-19

\begin{tabular}{|c|c|c|c|}
\hline $\begin{array}{c}\text { Autor / } \\
\text { País / } \\
\mathbf{N}^{\circ} \text { de pacientes }\end{array}$ & $\begin{array}{l}\text { Ambulatorios / } \\
\text { Hospitalizados }\end{array}$ & $\begin{array}{l}\text { Enfermedad autoinmune } \\
\text { ( } N^{\circ} \text { casos hospitalizados) }\end{array}$ & Fallecidos \\
\hline $\begin{array}{l}\text { Gianfrancesco et al. }{ }^{\text {a }} \\
\text { EE. UU. y países europeos } \\
548 \text { pacientes }\end{array}$ & $271 / 277$ & $\begin{array}{l}\text { Artritis reumatoide (104) } \\
\text { Lupus eritematoso sistémico (48) } \\
\text { Artritis psoriásica (22) } \\
\text { Espondiloartritis (16) } \\
\text { Vasculitis (24) } \\
\text { Otras (63) }\end{array}$ & $55^{*}$ \\
\hline $\begin{array}{l}\text { Pablos et al. }{ }^{b} \\
\text { España } \\
228 \text { pacientes }\end{array}$ & $-/ 228^{+}$ & $\begin{array}{c}\text { Artritis reumatoide (65) } \\
\text { Espondiloartritis (35) } \\
\text { Artritis psoriática (36) } \\
\text { Polimialgia reumática (24) } \\
\text { Lupus eritematoso sistémico (16) } \\
\text { Esclerosis sistémica (13) } \\
\text { Otros (39) }\end{array}$ & $41^{*}$ \\
\hline $\begin{array}{l}\text { Ye et al. }{ }^{c} \\
\text { China } \\
21 \text { pacientes }\end{array}$ & $0 / 21$ & $\begin{array}{l}\text { Artritis reumatoide (8) } \\
\text { Lupus eritematoso sistémico (4) } \\
\text { Síndrome de Sjögren (3) } \\
\text { Espondilitis anquilosante (2) } \\
\text { Otros (4) }\end{array}$ & $\begin{array}{l}2 \\
0 \\
0 \\
0 \\
0\end{array}$ \\
\hline $\begin{array}{l}\text { Fredi et al. } \\
\text { Italia } \\
65 \text { pacientes }\end{array}$ & $18 / 47$ & $\begin{array}{c}\text { Artritis reumatoide (16) } \\
\text { Espondiloartritis o artritis psoriásica (7) } \\
\text { Lupus eritematoso sistémico (3) } \\
\text { Esclerosis sistémica (3) } \\
\text { Otras (18) }\end{array}$ & $\begin{array}{l}4 \\
3 \\
1 \\
1 \\
1\end{array}$ \\
\hline $\begin{array}{l}\text { Haberman et al. } \\
\text { EE. UU. } \\
59 \text { pacientes }\end{array}$ & $45 / 14$ & $\begin{array}{c}\text { Artritis reumatoide (6) } \\
\text { Artritis psoriásica (3) } \\
\text { Colitis ulcerativa (3) } \\
\text { Otras (2) }\end{array}$ & $\begin{array}{c}0 \\
0 \\
0 \\
1\end{array}$ \\
\hline $\begin{array}{l}\text { Mathian et al. } .^{\dagger} \\
\text { Francia } \\
17 \text { pacientes }\end{array}$ & $3 / 14$ & Lupus eritematoso sistémico (17) & 2 \\
\hline $\begin{array}{l}\text { Moiseev et al. } \\
\text { Rusia } \\
10 \text { pacientes }\end{array}$ & $0 / 10$ & $\begin{array}{l}\text { Artritis reumatoide (5) } \\
\text { Artritis psoriásica (1) } \\
\text { Esclerosis sistémica (2) } \\
\text { Lupus eritematoso sistémico (1) } \\
\text { Espondiloartritis (1) }\end{array}$ & $\begin{array}{l}2 \\
0 \\
2 \\
0 \\
1\end{array}$ \\
\hline $\begin{array}{l}\text { Gartshteyn et al. } \\
\text { EE. UU. } \\
10 \text { pacientes }\end{array}$ & $3 / 7$ & Lupus eritematoso sistémico (7) & 0 \\
\hline $\begin{array}{l}\text { Lagadinou et al. } \\
\text { Grecia } \\
10 \text { pacientes }\end{array}$ & $3 / 7$ & $\begin{array}{l}\text { Esclerosis múltiples (3) } \\
\text { Artritis reumatoide (2) } \\
\text { Artritis psoriásica (1) } \\
\text { Tiroiditis de Hashimoto (1) }\end{array}$ & 0 \\
\hline $\begin{array}{l}\text { Cheng et al. } \\
\text { China } \\
5 \text { pacientes }\end{array}$ & $0 / 5$ & $\begin{array}{l}\text { Artritis reumatoide (4) } \\
\text { Esclerosis sistémica (1) }\end{array}$ & $\begin{array}{l}0 \\
0\end{array}$ \\
\hline $\begin{array}{l}\text { Monti et al. } \\
\text { Italia } \\
4 \text { pacientes }\end{array}$ & $3 / 1$ & Artritis reumatoide (1) & 0 \\
\hline
\end{tabular}

a Gianfrancesco M et al. Ann Rheum Dis. 2020:79(7):859-866. b Pablos Jl et al. Ann Rheum Dis. 2020:0:1-6. c Ye C et al. Ann Rheum Dis. 2020·79(8):1007-1013. d Fredi M et al. Lancet. 2020;2(9):e549-e556. ${ }^{e}$ Haberman R et al. N Engl J Med. 2020;383(1):85-88. ${ }^{f}$ Mathian A et al. Ann Rheum Dis. 2020;79(6):837-839. ${ }^{8}$ Moiseev S et al. Ann Rheum Dis. 2020;0:1-2. h Gartshteyn Y et al. Lancet. 2020; 2(8):452-454. ' Lagadinoua M et al. J Clin Med Res. 2020;12(8):539-541. ' Cheng C et al. Clin Rheumatol. 2020; 39(7):2025-2029. ${ }^{k}$ Monti S et al. Ann Rheum Dis. 2020;79(5):667-668.

* El estudio no especifica el tipo de enfermedad autoinmune y los casos de mortalidad.

+ Estudio observacional retrospectivo de cohortes emparejadas en el que se comparó pacientes hospitalizados con enfermedades autoinmunes vs. pacientes hospitalizados sin enfermedades autoinmunes (no se conoce el número de pacientes con enfermedades autoinmunes y COVID-19 tratados ambulatoriamente). 
Una explicación podría ser la inmunomodulación farmacológica (DMARD, terapia biológica, inhibidores JAK) utilizada en las enfermedades autoinmunes, que disminuiría la probabilidad de desarrollar la denominada "tormenta de citoquinas", la cual jugaría un rol crucial en el desarrollo de la forma severa de la infección y en el subsecuente riesgo de mortalidad por COVID-19.

Si bien en el contexto de coexistencia de una infección y enfermedad autoinmune, la indicación es la suspensión temporal del tratamiento inmunomodulador, para la infección por COVID-19 esto podría ser diferente, y estos fármacos inmunomoduladores (a dosis adecuadas) podrían ser claves en el tratamiento y recuperación de los mismos ${ }^{[5]}$.

Sin embargo, esto aún es una hipótesis que debe ser estudiada y bajo ninguna circunstancia se debería dejar de lado las estrictas medidas de prevención y cuidado en estos pacientes, tal como lo aconseja el Colegio Americano de Reumatología y diversos autores ${ }^{[6,7]}$. Es necesario que, se realicen más estudios donde se pueda dilucidar el comportamiento de la COVID-19 en las enfermedades autoinmunes y el impacto en la morbimortalidad de este grupo de pacientes.

Contribuciones de autoría: todos los autores han participado en la concepción y diseño del artículo, así como en la aprobación de la versión final. EZF y JSM participaron en la recolección, análisis e interpretación de datos y en la redacción del artículo

Potenciales conflictos de interés: ninguno declarado por los autores.

Fuentes de financiamiento: autofinanciado.

\section{ORCID}

Ernesto Zavala Flores, https://orcid.org/0000-0002-7389-5576 Jannin Salcedo Matienzo, https://orcid.org/0000-0002-9221-3040 Alfredo Berrocal Kasay, https://orcid.org/0000-0001-8640-6658

\section{REFERENCIAS BIBLIOGRÁFICAS}

1. Sanyaolu A, Okorie C, Marinkovic A, Patidar R, Younis K, Desai P, et al. Comorbidity and its Impact on Patients with COVID-19. SN Compr Clin Med. 2020;1-8. doi:10.1007/s42399-020-00363-4.

2. Fredi M, Cavazzana I, Moschetti L, Andreoli L, Franceschini F, Airò $P$, et al. COVID-19 in patients with rheumatic diseases in northern Italy: a single-centre observational and case-control study. Lancet. 2020;2(9):E549-E556. doi: 10.1016/S2665-9913(20)30169-7.

3. Ehrenfeld M, Tincani A, Andreoli L, Cattalini M, Greenbaum A, Kanduc D, et al. Covid-19 and autoimmunity. Autoimmun Rev. 2020;19(8):102597. doi: 10.1016/j.autrev.2020.102597.

4. Emmi G, Bettiol A, Mattioli I, Silvestri E, Di Scala G, Urban ML, et al. SARS-CoV-2 infection among patients with systemic autoimmune diseases. Autoimmun Rev. 2020;19(7):102575. doi: 10.1016/j. autrev.2020.102575.

5. Lu C, Li S, Liu Y. Role of immunosuppressive therapy in rheumatic diseases concurrent with COVID-19. Ann Rheum Dis. 2020;79(6):737739. doi: 10.1136/annrheumdis-2020-217460.

6. Nueva guía clínica del Colegio Americano de Reumatología para el tratamiento de pacientes de reumatología durante la crisis de COVID-19 [Internet]. CREAKYJOINTS. 2020 [citado el 6 de junio de 2020] Disponible en: https://creakyjoints.org.es/2020/04/20/nueva-guiaclinica-del-colegio-americano-de-reumatologia-para-el-tratamientode-pacientes-de-reumatologia-durante-la-crisis-del-covid-19/

7. Grange L, Guilpain P, Truchetet ME, Cracowski IL, Société française de pharmacologie et de thérapeutique (SFPT). Challenges of autoimmune rheumatic disease treatment during the COVID-19 pandemic: A review. Therapie. 2020;75(4):335-342. doi: 10.1016/j.therap.2020.06.013. 